\title{
Survey of Media Access Control (MAC) and Routing Technologies of WiFi-MESH wireless Network
}

\author{
ZHU Shibing ${ }^{1,}$, DAI Jianmei, b \\ ${ }^{1}$ Department of Information Equipment, Academy of Equipment, Beijing, China \\ a13910953181@139.com, benxuelan0402@126.com
}

\section{Keywords: WiFi-MESH;802.11;CSMA/CA;TDMA;HWMP;BATMAN-adv}

Abstract. WiFi-MESH wireless network which are mainly based on the 802.11 protocols are developed quickly in recent years. The principle features, difficulties, directions and application schemes of the key technologies of WiFi-MESH systems, including MAC mechanisms and routing protocols, are analyzed and discussed. It can be concluded in the paper that the TDMA mechanisms based on 802.11 should be developed further for avoiding the collisions in essence, and the new BATMAN-adv routing protocol based on OSI architecture layer two may raise the performance of the systems more significantly than the HWMP routing protocol. Finally, some new technologies which could be used in WiFi-MESH are discussed.

\section{Introduction}

WiFi-MESH Wireless Network are mainly used for the aircrafts networking, vehicles networking, smart-cities, emergency communications and the tactical communication in the battlefield, due to its combination of characteristics of self-organized, self-mended, self-meditated and multi-hop relay of ad hoc network and the advantages of high intensity broadband, easy access and connection of traditional WiFi technology. Many systems have already been produced, such as Strix systems which is owned by Strix Corporation in USA, and "W-MESH Wireless Network" which is owned by Equipment Academy in China. In spite of this, there are a great quantity of in depth experiments ought to be conducted for the higher demand on data rate, fast connection between networks and systems, and efficiency of frequency spectrum and anti-interference ability, as the accelerating development on the information technology, and the continuous need of larger capacity and higher data rate by users.

Media Access Control (MAC) and network routing technology are two key technologies of WiFi-MESH wireless network. The principle features, difficulties, directions and application scheme of the key technologies of the WiFi-MESH systems are analyzed and discussed in the paper.

\section{MAC Layer Mechanism}

MAC Layer mechanism of 802.11 is based on CSMA/CA Mechanism conventionally. The CSMA/CA mechanism is simple and stable in the low loading network, while it may lead to node collision, unfairness, unpredictable and unstable problems in the high loading network. Scholars have provided a lot of approaches of improvement about CSMA/CA, but these approaches cannot solve the above problems in essence. From another opinion, some scholars introduce mechanisms of TDMA to Protocol 802.11, which could guarantee transmission of data without collision effects, to make systems adapt to outdoor environment and long-range multiple hop transmission.

\section{Improvement of the Fairness of CSMA/CA Mechanism}

1) The Contention Window Adjustment Method ${ }^{[1]}$.

The method can raise the capacity in long term by banning reset value of contention window reaching the least, but it fails to consider the short term fairness which makes some of the nodes suffer continuous collision and be forced to stay in the period of backoff for a long time.

2) Adjustment of Competitive Parameter ${ }^{[2]}$.

This method can raise the capacity and gives consideration to fairness by the estimation of competitors (users) and adjustment of competition parameter. However, it complicate the 
communication, and would greatly downgrade the accuracy of estimation mentioned above especially when errors appear during such process.

3) Deterministic Backoff.

This kind of methods turn the random mechanism of backoff into some definite mechanism mechod to almost reach non-collision transmission. Literature [3] and [4] put forward a method (CSMA/ECA) for deterministic backoff; Literature [5] considers fairness and the multi-hop character further, but the users in the system can not exceed the fixed quantity due to the deterministic backoff level; Literature [6] puts forward a method of sluggishly deterministic backoff. These methods mentioned can increase the capacity of the system through modification of CSMA/ECA, and assure further long-term fairness in the process of communication with the combination of fair-share strategy.

At present, some methods have been implemented in software and hardware, and they are expected to be accepted by the 802.11 standards. However, it is worth considering that the methods mentioned above mainly consider backoff mechanisms of CSMA/CA and fairness issues, they cannot fundamentally solve the problem of data collision, and are limited to enhance throughput capacity in case of large capacity users.

\section{TDMA Improvement Technology Based on 802.11}

Moraes $^{[7]}$ proved the feasibility of introducing TDMA technology to 802.11 system firstly, and ROSALNet $^{[8]}$, roadside Mesh network ${ }^{[9]}$, Driver level implementation of TDMA MAC in long distance $\mathrm{WiFi}^{[10]}$ and other actual cases of application have further proved that, there are lower delay and jitter, and more robust characteristics in the WiFi-Mesh Wireless Network based on TDMA, and it is more suitable for longer distance and provides better support for mobility. The difficulty of implementation of TDMA lies on synchronization accuracy problem. Djukic ${ }^{[11]}$ put forward a protocol named Soft-TDMAC, which realizes synchronization amid nodes via phase-locked loop. It realizes intense synchronization, downgrades errors and upgrades accuracy and allocation efficiency in the whole network through establishing synchronization tree with minimum hops in the whole network, but its reliability is low. Literature [12] discusses the theory of LiT-MAC and the procedure of implementation. This method raises the reliability of synchronization and take considerations of transmission with long distance and multichannel. However, the capacity of anti-interference is relatively low. Moreover, it needs united and concentrated supervisors for time scheduling. From a practical point of view, the current common practice to support the TDMA is based on modifying 802.11 protocol, namely these systems cannot communicate with other 802.11 systems seamlessly, and the system flexibility is reduced. The versatility of system will be significantly improved if the Soft-ware-Defined Networking (SDN) was adopted and a united layer was added for control to implement TDMA without changing the original structure. In addition, the timing accuracy will be raised if Precise Time Protocol (PTP) and some new technologies were utilized.

\section{CSMA/TDMA Combination Technology}

The combination of CSMA and TDMA is another way, and it is a coordinated consideration balancing both complexity and performance which is more suitable for multi-hop networks with less node. Sayadi ${ }^{[13]}$ put forward one called one shot Slot TDMA-based Reservation (OSDR), which divide a time frame into two sub frames. One is CONTROL sub frame which is complied with CSMA/CA channel, and it is mainly in charge of short messages transmission with less volume of data like control order. The other is DATA sub frame which is in accordance with fixed scheduling mode, and it is in charge of operation data. This method not only makes use of fixed time-slot allocation of TDMA, but also realizes dynamic on-demand time-slot allocation of CSMA throughout the whole networks.

The above method increases bandwidth of the system and is very suitable for multi-hop transmission, but it doesn't consider the QoS of service. Literature [14] proposes a continuous collision free MAC adaptive algorithm UCFA using a deterministic backoff method combined with fixed TDMA time slot scheduling strategy, it can guarantee a collision free data transmission without the need to consider the flow type and the number of terminals. 


\section{Routing protocols}

Routing protocols are crucial to realize multi-hop WiFi-MESH wireless network system. Routing protocols are mainly divided into two types. One is $3^{\text {rd }}$-layer routing protocol based on network layer, and the other is $2^{\text {nd }}$-layer routing protocol based on link layer. The $2^{\text {nd }}$-layer routing protocols needn't frequently read, write and exchange data between space of users and kernel comparison with 3-layer routing protocol, and can decreases the cost of handling database greatly. Besides of that, transparency of network layer and expansibility of protocols are grandly raised. The representative two $2^{\text {nd }}$-layer routing protocols are Hybrid Wireless MESH Protocol(HWMP) and Better Approach To Mobile Ad-hoc Networking Advanced(BAT-MAN-adv) routing protocols.

\section{HWMP Routing Protocol and Its Improvement Technologies}

HWMP protocol is a comprehensive protocol which is developed by 802.11 s Task Group for WiFi-Mesh network. This protocol absorbs the advantages of both reactionary routing protocol and proactive routing protocol based on tree topology, which is more compatible with Wireless Mesh Network. The current improvement policies mainly optimize property of system through different routing metrics as follows: Reservation-based HWMP (R-HWMP ${ }^{[15]}$ protocol upgrade Quality of Service through introducing fields of RSpec and TSpec; HWMP-ETX ${ }^{[16]}$, Q-HWMP ${ }^{[17]}$ and HWMP+ ${ }^{[18]}$ protocols reduce the delay and packet loss probability through introducing Expected Transmission count, and delay, link quality, throughput and other routing metrics; Eltahir and other scholars ${ }^{[19]}$ introduce a Link Expiration Time(LET for short) into routing metrics taking advantage of stability of LET decisive link, which can increase mobile adaptive ability. In addition, energy-efficient HWMP protocol $^{[20]}$ strengthen energy validity through making surplus energy of nodes as routing metrics. Improvement policies mentioned above can raise up QoS, time delay, throughput of information, energy validity and other properties to some extent. However, just as the traditional protocols, they are lacking in valid strategies of congestion control and don't fully consider load balancing, and the traffic overload of root nodes in networks would appear if there is a large amount of data waiting for transmission.

\section{BATMAN-adv Routing Protocol and Its Improvement Technologies.}

BATMAN-adv is a new routing protocol introducing collective intelligence. The basic idea is to counter the boundary effect caused by the network fluctuation and compensate the instability, the new aspect is that the network topology information is maintained by all nodes in the network, and it is very suitable to be applied on the instable wireless mesh network. Many scholars have done practical tests ${ }^{[21-23]}$ on this routing protocol and compared it with other open source routing protocols. The analysis of such tests proves that this arithmetic performs well in packet loss rate, time delay and network throughput and owns better stability in comparison with HWMP ${ }^{[24-25]}$. However, this routing protocol has the problem of slow convergence after the change of the network topology ${ }^{[26-27]}$. Improvement schemes are listed as follows:

(1) Optimizing Sending Interval of Originator Message

The default setting of OGM sending interval is 1 seconds. Reducing the interval time can speed up the path finding, but it can reduce the bandwidth of the two terminal. It has been found that when sending interval is set to 0.2 seconds ${ }^{[28]}$, the tradeoff between convergence and bandwidth would be established.

(2) Optimizing Slide Window Mechanism

Researchers have found that when the latest serial number of this node has not been completed, if the OGM message is received, the difference between the OGM message and the latest serial number recorded by the node of the node will be calculated to generate error in the window range, and slide window would reset and go into protection period and abandon OGM messages. This phenomenon lowers the rate of convergence. Literature [29] adds up a switch in charge of checking nodes and the list of local neighborhood. When the list is vacant, close the window protection mechanism; when new neighbors exist, open the mechanism. This method avoid the delay caused by renewing lists of source nodes and raises up the convergence speed of routing process. 
(3) Optimizing Transmit Quality

The fast convergence can be achieved by improving the local TQ, transmission TQ and global TQ calculation method ${ }^{[30-31]}$. In addition, the convergence speed can also be optimized by improving the signal strength and improving the message processing mechanism.

\section{The Further prospect of above technologies}

(1) CSMA and TDMA mechanisms have different advantages. The need can be satisfied and universality and scalability of the system can be maintained by optimizing CSMA under the Scenarios of low load, non-real time and near transmission distance; on the other hand, TDMA is more useful to increase performances of the system with high load, long transmission distance and high real-time requirement, but we need to consider the complexity and synchronization precision; third, the combination of CSMA and TDMA can obtain the balance between complexity and performance of systems which is multi-hop but with little nodes.

(2) It is the priority in the future to adopt $2^{\text {nd }}$-layer routing protocol. From the practical view, though HWMP is the standard routing protocol of 802.11 s, it is far from actual application and deployment; the published Open Source BATMAN-adv protocol, with its advanced designing ways of lightweight, inter-platform and routing searching strategies makes it with a brighter prospect. It is a very pragmatic protocol under the premise of the fundamental optimization of the convergence speed.

\section{Conclusions}

The cellular communication system, broadband wireless access system, and other wireless networks are developing rapidly with the continuous progress of information technology. The 802.11 WiFi-MESH wireless network will also be developed by leaps and bounds. For instance, the resources (optimizing channel capacity and spectrum sharing) management will be more efficient through introducing coordinated communication technique like "user cooperative diversity" technology; the spectrum utilization efficiency will be raised if the recognize radio is introduced; the rate of abnormal link interrupt and message lost with important messages will be decreased by introducing Content Cache Technology; the message transmission will be more robust by introducing Delay Tolerant Network Technology, even links are fluctuating.

\section{References}

[1] WANG C, LI B, LI L. A new collision resolution mechanism to enhance the performance of IEEE802.11 DCF [J]. IEEE Transactions on Vehicular Technology, 2004, 53 (4):1235-1246

[2] TOLEDO A L, VERCAUTEREN T, WANG X. Adaptive optimization of IEEE802.11 DCF based on Bayesian estimation of the number of competing terminals [J]. IEEE Transactions on Mobile Computing, 2006, 5(9):1283-1296

[3] BARCELO J, BELLALTA B, SFAIROPOULOU A, et al. CSMA with enhanced collision avoidance: a performance assessment [C]. //IEEE Vehicular Technology Conference. Barcelona: IEEE, 2009:1-5.

[4] HE Y, YUAN R, SUN J, et al. Semi-random backoff: towards resource reservation for channel access in wireless LANs[C]. $/ / 17^{\text {th }}$ IEEE International Conference on Network Protocol. Princeton: IEEE, 2009:21-30

[5] HUI K, LI T, GUO D, et al. Exploiting peer to peer state exchange for distributed medium access control [C]. //IEEE International Symposium on Information Theory Proceedings (ISIT). Petersburg: IEEE, 2011:2368-2372. 
[6] SANABRIA-RUSSO L, FARIDI A, BELLALTA B, et al. Future evolution of CSMA protocols for the IEEE 802.11 standard[C]. //IEEE International Conference on Communications. Budapest: IEEE, 2013:1274-1279.

[7] MORAES R, VASQUES F, PORTUGAL P. A TDMA based mechanism to enforce realtime behavior in WiFi networks factory communication systems[C]. //IEEE International Workshop on Factory Communication Systems. Dresden: IEEE, 2008:109-112.

[8] RAKHEJA N, BHATIAP. ROSALNet: a spectrum aware TDMA Mesh network for rural internet connectivity communications[C]. $/ / 20^{\text {th }}$ National Conference on Communications. Kanpur: IEEE, 2014:1-6.

[9] SAFRONOV R, BAKHTIN A. Designing roadside Mesh network with TDMA [C]. $/ / 6^{\text {th }}$ International Congress on Ultra-Modern Telecommunications and Control Systems and Workshops (ICUMT). Petersburg: IEEE, 2014:131-135.

[10] AHMED S S, HUSSAIN I, AHMED N. Driver level implementation of TDMA MAC in long distance WiFi [C]. //International Conference on Computational Intelligence and Networks (CINE). Bhubaneswar: IEEE, 2015:80-85.

[11] DJUKIC P, MOHAPATRA P. Soft-TDMAC: a software-based 802.11 overlay TDMA MAC with microsecond synchronization [J]. IEEE Transactions on Mobile Computing, 2012, 11(3):478-491.

[12] SEVANI V, RAMAN B, JOSHI P. Implementation-based evaluation of a full-fledged multihop TDMA-MAC for WiFi Mesh networks [J]. IEEE Transactions on Mobile Computing, 2014, 13(2):392-406.

[13] SAYADI A, WEHBI B, LAOUITI A. One shot slot TDMA-based reservation MAC protocol for wireless ad hoc networks[C]. //IEEE 73rd Vehicular Technology Conference (VTC Spring), Yokohama, IEEE, 2011:1-5.

[14] TUYSUZ M F, MANTAR H A. An uninterrupted collision-free channel access scheme over IEEE 802.11 WLANs[C]. //IEEE Wireless Communications and Networking Conference (WCNC). Shanghai: IEEE, 2013:386-391

[15] KIM B C, CHOI H S, MIN S H, et al. R-HWMP: reservation-based HWMP supporting end-to-end QoS in wireless Mesh Networks[C]. //2013 International Conference on Information Networking (ICOIN). Bangkok: IEEE, 2013:385-390.

[16] NARAGUND J G, BANAKAR R M. Analysis of HWMP-ETX Routing in Wireless Mesh networks advanced computing[C]. //2013 2nd International Conference on Networking and Security (ADCONS). Mangalore: IEEE, 2013:208-213.

[17] BEN-OTHMAN J, MOKDAD L, CHEIKH M O. Q-HWMP: improving end-to-end QoS for 802.11s based Mesh networks[C]. //IEEE Global Telecommunications Conference (GLOBECOM 2010). Miami: IEEE, 2010:1-6.

[18] YANG L H, CHUNG S H. HWMP+: an improved traffic load scheme for wireless Mesh networks[C]. //2012 IEEE 14th International Conference on High Performance Computing and Communication \&2012 IEEE 9thInternational Conference on Embedded Software and Systems (HPCC-ICESS). Liverpool: IEEE, 2012:722-727.

[19] ELTAHIR A A, SAEED R A, ALAWI M A. An enhanced hybrid wireless Mesh protocol (E-HWMP) protocol for multihop vehicular communications[C]. //2013 International Conference on Computing, Electrical and Electronics Engineering (ICCEEE). Khartoum: IEEE, 2013:1-8. 
[20] MING ANN, YAU K L A. An energy-efficient hybrid wireless Mesh Protocol (HWMP) for IEEE 802.11s Mesh networks[C]. //IEEE International Conference on Control System, Computing and Engineering (ICCSCE). Mindeb: IEEE, 2013:17-21.

[21] BAROLLI L, IKEDA M. Performance analysis of OLSR and BATMAN protocols considering link quality parameter[C]. //Proceedings of International Conference on Advanced Information Networking and Applications. Bradford: IEEE, 2009:307-314.

[22] CHISSUNGO E, BLAKE E, LE H. Investigation into BATMAN-adv protocol performance in an indoor Mesh potato testbed $[\mathrm{C}]$. //Proceedings of Conference on Intelligent Networking and Collaborative Systems. Fukuoka: IEEE, 2011:8-13.

[23] ANAS N M, HASHIM F K. Performance analysis of outdoor wireless Mesh network using B.A.T.M.A.N.advanced [C]. //16th IEEE/ACIS International Conference on Software Engineering, Artificial Intelligence, Networking and Parallel/Distributed Computing (SNPD). Takamatsu: IEEE, 2015:1-4.

[24] NXUMALO S, NTLATLAPA N. Performance evaluation of routing metrics for community wireless Mesh networks [C]. //17th International Conference on Intelligent Sensors, Sensors Networks and Information Processing (ISSNIP). Adelaide: IEEE, 2011:556-561.

[25] SEITHER D, KONIG A, HOLLICK M. Routing performance of wireless Mesh networks: a practical evaluation of BATMAN advanced[C]. //IEEE 36th Conference on Local Computer Networks. Bonn: IEEE, 2011:897-904.

[26] GARROPPO R G, GIORDANO S, TAVANTI L. Experimental evaluation of two open source solutions for wireless Mesh routing at layer two [C]. //5th IEEE International Symposium on Wireless Pervasive Computing. Modena: IEEE, 2010:232-237.

[27] VADIM G, OLEG G, LEONID K, et al. An experimental comparison of dynamic routing protocols in mobile networks[C]. //IEEE 11th International Conference on Informatics in Control, Automation and Robotics (ICINCO).Vienna: IEEE, 2014(2):775-782.

[28] CHEN Yu. Research of switch mechanisms of Wireless Mesh Network based on BATMAN-adv main railroad. Changsha, Central South University, 2014:43-52.

[29] SHEN Shuang, LI Zhaowen, LUO Jun. Analyses and Optimization towards BATMAN-adv Routing Protocol of Wireless Mesh Network [J]. Microcomputer Information, 2012(10):327-329

[30] GAO Jian. Optimization and research for BATMAN Routing Protocol of Wireless Mesh Network [D]. Wuhan, Wuhan University of Technology. 2013:22-51

[31] ZHANG Tianyu. An improvement method based on batman's Mesh routing protocols [D]. Dalian, Dalian University of Technology 2014:22-51 